\title{
Globalización y formación profesional: ¿Nuevas reglas?
}

\author{
Teresita Durán Ramos
}

\begin{abstract}
Resumen: En el pasado, adquirir una formación profesional representaba la posibilidad de obtener empleo formal en el área respectiva y el ascenso casi garantizado a lo largo de la vida, con frecuencia dentro de una misma institución. El conocimiento profesional resultaba duradero.

El avance tecnológico y la economía globalizada han generado nuevas reglas del juego que constituyen en sí mismas, potencialmente, oportunidad y riesgo ${ }^{1}$.

En las nuevas reglas del juego derivadas del contexto económico global, del énfasis en la competitividad y del acceso directo al vertiginoso avance tecnológico, cada vez se dispone menos de la continuidad laboral, que suponía el estudiar una profesión.

Me parece que nuevamente la educación tiene sobre sí los reflectores. Si bien es cierto que las reglas del juego han cambiado, tanto desde el punto de vista económico como también en cuanto a las posibilidades que hoy día proporciona la información, las necesidades humanas fundamentales siguen siendo las mismas. La siguiente ponencia presenta una reflexión acerca del fenómeno de la globalización y su incidencia en la Educación Superior. Cuestiona la tarea de la Universidad en el contexto del avance tecnológico y la economía globalizada.
\end{abstract}

\section{Introducción}

El juego ha comenzado, afecta a todos los órdenes: económico, tecnológico, cultural. Podemos ganar o perder, lo único que no podemos evitar es participar. El juego se llama globalización.

La óptica que me conduce hacia la relación de este tema con el de la formación profesional es la que resulta de ser pedagoga y dedicar a la docencia universitaria gran parte de mis horas de trabajo.

Desde diversas fuentes se emite información acerca del impacto originado por la globalización. Se han acuñado, recientemente, barbarismos tales como "globalifóbico" y "globalifílico", pretendiendo con ello esquematizar de manera rígida un asunto de tan amplias y variadas consecuencias y reducirlo a un tablero de blanco y negro. La realidad no es, para bien y para mal, algo tan simple.

Existen efectos de la globalización, a mi parecer, tanto benéficos como perjudiciales. En el aspecto de la educación en general y del nivel superior en particular, el crecimiento exponencial de las posibilidades de comunicación de información y la velocidad con la que es posible acceder a ésta, resulta sin duda una ventaja imposible 
de ignorar. Las nuevas tecnologías derivadas de los progresos de la telefonía, la red, el uso del tablero en tiempo real, la televisión por cable, el video y en general los medios interactivos, configuran una cultura del aprendizaje permanente.

Algunos de los más importantes retos de la educación superior versan hoy en torno a cómo lograr atender la demanda sin disminuir más la calidad; en cómo democratizar el acceso a la educación sin caer en populismos; en cómo convertir en rentables los procesos de formación profesional sin abaratar sus resultados.

La tecnología por sí sola, por más avanzada que se encuentre, no sirve para atender y resolver problemas mal definidos o incorrectamente enfocados. El uso de los adelantos producidos por la más alta tecnología sin un sentido de responsabilidad, sin un proyecto educativo humanista $\mathrm{y}$ tendiente a la equidad y a la solidaridad, lleva a que lo que ya se hace mal, se siga haciendo peor, sólo que más rápido.

Así, el énfasis debe estar dado por reconocer que la gente va primero, que la pedagogía no puede quedar al servicio de la tecnología, a merced de los embates de la competencia o del mercado.

\section{La formación profesional tradicional}

El parteaguas que representó la posguerra, como todos sabemos, originó que la educación superior en nuestro país después de la primera mitad del siglo XX, respondiera a la necesidad de formar cuadros profesionales que apoyaran la construcción moderna y el desarrollo tanto tecnológico como industrial y empresarial de México. Tal fenómeno encontró similitud con lo ocurrido, en distintos momentos, en un gran número de sociedades occidentales tanto del continente americano como del europeo.

Durante estos años, adquirir una formación profesional representaba la posibilidad de obtener empleo formal en el área respectiva y el ascenso casi garantizado a lo largo de la vida, con frecuencia dentro de una misma institución. El conocimiento profesional resultaba, entonces, duradero.

De este modo, aunque no todos los individuos alcanzaran este ideal, el conocimiento y la especialización depositados en algunos de sus miembros, representó también la posibilidad de fortalecer las relaciones con la comunidad de base. La movilidad social que la carrera profesional generalmente promovió, actuaba como un factor de reconocimiento social y apuntaba hacia la legitimidad del sistema. Así, la carrera profesional constituyó por mucho tiempo un fundamento económico, ideológico y social para amplios sectores, por su carácter de medio comprobado para llevar a la realización el proyecto de vida que prometía. Parecía que bastaba ser ingeniero, médico, contador, abogado, etc., para asegurarse una posición económica a través del desempeño laboral esperado, junto con otros profesionales, pero sobre todo para adquirir una identidad tanto personal como social.

En las últimas décadas, el avance tecnológico y la economía globalizada han generado para el mundo del trabajo nuevas reglas del juego que constituyen en sí mismas escenarios inéditos, y por ello significan, potencialmente, tanto riesgo como oportunidad $^{2}$.

\section{Nuevas reglas}

Las reglas han cambiado no sólo para beneficio. Hablando de progreso tecnológico, en el campo del desempeño laboral, el 
impacto ha sido trascendental. Derivadas del contexto económico global, las nuevas reglas del juego cuyo énfasis está dado en la mayor competitividad y en el acceso directo al vertiginoso avance tecnológico y su aplicación en las más diversas áreas, el efecto ha sido que cada vez se dispone menos de la continuidad laboral, que implicaba el estudiar una profesión.

Como una sola de las consecuencias derivadas de esta transformación económica, tecnológica y de progresiva competencia, la reingeniería de procesos, por ejemplo, ha llevado a eliminar capas completas de actividad laboral debido a que los sistemas de procesamiento electrónico de información hacen posible una coordinación mucho más eficaz, con un menor número de personas, para el logro de los fines de una organización de cualquier ramo ${ }^{3}$.

Los proyectos de reestructuración han ido avanzando desde los procesos en particular hasta las organizaciones en general. En los últimos años hemos sido testigos de la desaparición de grandes empresas que fueron literalmente tragadas por otras, las cuales no necesariamente cuentan ahora con mayor número de empleados. Las fusiones, de igual modo, respondieron y responden a procesos distintos diseñados para alcanzar las mismas metas a un costo más bajo. Los empleos tradicionales han sido sustituidos por la contratación de consultores externos y profesionales que ofrecen sus servicios de manera independiente. Todo esto ha impactado y seguirá impactando directamente sobre los modos usuales de desempeño y permanencia laboral.

Los cambios recientes en ese aspecto se han originado por el rediseño de procesos y productos en función de la búsqueda de la calidad, de manera conjunta con la reducción de los dispendios de toda índole. Es decir, que cuando el discurso político habla de abatir el índice de desempleo en nuestro país, seguramente no está siquiera soñando con restablecer el número de empleos perdidos tal como existían o reinstalar a las personas en puestos equivalentes a los que tuvieron en el pasado.

Las condiciones económicas y su obvia derivación hacia la demanda de profesionales han traído como consecuencia el hecho de que se observe, en organizaciones de diversa índole, una marcada tendencia hacia los pagos en función directa del desempeño.

Además de lo anterior, como resultado de una nueva integración de factores tanto económicos como políticos y culturales, las comunidades de trabajo se están transformando y han dejado de ser socialmente cercanas. Muchas de las redes laborales que se constituyen en la actualidad, implican una paradoja, debido a la asombrosa reducción del tiempo necesario para establecer el contacto con quienes se encuentran a grandes distancias, físicamente hablando, y la facilidad de asegurar una comunicación con ellos, frente a la ausencia o superficialidad en la relación personal con quienes comparten en su diaria jornada laboral, las mismas instalaciones: es fácil comunicarnos con quienes se encuentran distantes y difícil con los más próximos.

Como consecuencia, la capacidad de apropiarse y dominar los conocimientos profesionales que se alcanzaba a lo largo de toda una vida, ha sido sustituida por la urgencia de conseguir adaptarse, de manera constante, a nuevos y cambiantes contextos sociales sin verse afectado por ello.

No es una exageración decir que en sociedades como las que nos está tocando vivir, lo único permanente es el cambio. Así, las formaciones profesionales especializadas tal como han sido concebidas en el pasado, 
tenderán a caracterizarse por un periodo de vida laboral mucho más corto, debido a la consecuente pérdida acelerada de vigencia de los conocimientos adquiridos.

\section{Retos actuales de la formación profesional}

La superespecialización que había venido generándose desde principios de siglo, ha resultado una arma de dos filos bien cortantes. En ocasiones se ha tendido a considerar que, ante tal estado de cosas, la tarea de la educación superior consistiría ahora en echar a andar los mecanismos para que los egresados sean capaces de desarrollar diversas destrezas que les permitan cambiar de actividad varias veces a lo largo de su vida; procurar que se formen "generalistas" en lugar de "especialistas" como se hizo en el pasado ${ }^{4}$.

¿Será ésta la única tarea de la educación profesional para nuestro tiempo? ¿Dónde quedaría entonces la formación humana dentro de esta urgencia de acelerar la respuesta adaptativa al mercado?

Me parece que de nuevo la educación de todos los grados, modalidades y niveles tiene sobre sí los reflectores. Si bien es cierto que las reglas del juego han cambiado, tanto desde el punto de vista económico como también en cuanto a las posibilidades que hoy día proporciona la información, las necesidades humanas fundamentales siguen siendo las mismas. Es decir, las necesidades de cada individuo y su futuro son tan importantes como las necesidades sociales y de la producción.

A través del trabajo profesional el individuo busca, además de la subsistencia: un reflejo, un principio de identidad. Busca también una vinculación con otros, satisfacer su sentido de pertenencia y a la vez reconocerse autónomo y útil a su comunidad.
Para que pueda lograrlo es necesario que su formación incluya la adquisición de habilidades cognitivas, reflexivas y críticas así como también la comprensión de la cultura en su conjunto: humanista, científica y tecnológica.

El hecho de que las condiciones actuales impongan cambios sensibles a los egresados de las instituciones que ofrecen estudios superiores, no debe tener como consecuencia el que la formación y la educación para el trabajo se descuiden.

Desde la educación es necesario hoy, más que nunca, fortalecer el sentido de identidad, de responsabilidad y de compromiso social de los individuos a partir de la búsqueda de la excelencia en el desarrollo de todas sus capacidades: la escuela tiene sobre sí la gran tarea de formar gente altamente asertiva; predispuesta a la generación de redes de comunicación interpersonal para imaginar y conducir el esfuerzo conjunto hacia soluciones novedosas; tendiente a estimularse frente al reto, convencida de la oportunidad de poder construir y construirse con otros, comprometida con el desarrollo económico, político y social de su comunidad.

Ya no existen más las condiciones de estabilidad en las que bastaba estudiar una profesión e insertarse en el campo de trabajo para encontrar, en la tarea y participación con otros, sentido a la construcción de lo individual y lo social. Si tuvieron o no razón de ser las prácticas educativas más tradicionales que todos conocemos, y que permitieron a los egresados ubicarse de manera predeterminada en la división social del trabajo, hoy en día no son útiles para que las personas logren responder a los nuevos retos ${ }^{5}$.

Por supuesto que en la formación de profesionales siempre fue bienvenida, además del dominio de los conocimientos 
probados, una importante dosis de imaginación para innovar y mejorar lo existente, el desarrollo de la capacidad de comunicación, la tendencia hacia el estudio y la actualización permanentes, la capacidad de integrarse a grupos de trabajo, etcétera ; sólo que hoy, el desarrollo de éstas y otras habilidades, se encuentra en el terreno de lo que debe ser considerado imprescindible.

Ha sido una preocupación presente en mi labor el hecho de promover el sentido de responsabilidad de los estudiantes en su propia formación y la valoración del autodidactismo como vía para alcanzar los niveles educativos más altos. La tarea del docente, así como la asignada a la organización institucional y a los sistemas de enseñanza, debe ser, desde esta perspectiva, la de constituirse en instancias promotoras, impulsoras y socializadoras del avance y desarrollo educativo de los alumnos.

Acorde con el avance evolutivo de los distintos estadios de madurez cognitiva, la tarea docente y la intervención institucional en su conjunto debieran mantener este papel de ser promotores del aprendizaje y la superación del estudiante, sin señalar caminos únicos ni límites máximos. Así, los procesos prioritarios que tendrían lugar en los distintos grados y niveles de la educación, serían los de creación, invención, descubrimiento, búsqueda del conocimiento e inquisitivos; en pocas palabras: procesos de auténtica formación.

Todo lo anterior parecería sólo reiteración de las diferencias que para algunos existen y han existido a lo largo de la historia de la educación entre los sistemas activos y los tradicionales; sin embargo, en lo que concierne al nivel de educación superior, enfatizar y recuperar ventajosamente las características del aprendizaje activo resulta, en este momento, fundamental.
Ya en alguna ocasión me he referido a la importancia de concebir de manera integral la formación profesional, poniendo un acento importante en la adquisición de actitudes de logro identificadas en el pasado como exclusivas de los líderes. De ese trabajo anterior 6 retraigo ahora los puntos más concretos que, a mi modo de ver, deben estar presentes en la educación superior:

- Fomentar en el estudiante la búsqueda de la información

- Instarlo a que llegue a su propio límite en cuanto al interés que el aprendizaje de un asunto le despierte

- Estimular al estudiante para que construya su propio saber

- Diseñar actividades de aprendizaje como guía, posibilidad e incentivo para la acción original, productiva y consciente del estudiante, sobre el conocimiento

- $\quad$ Propiciar en los alumnos una actitud inquisitiva y crítica

- Fomentar que los estudiantes sean capaces de construir planteamientos originales tendientes a resolver problemas reales

- $\quad$ Promover la realización de trabajos y proyectos escolares en los que se vierta un principio de crítica y aportación personal como producto de aprendizaje individual y grupal

- Propiciar experiencias o prácticas escolares reales en colaboración con instituciones, grupos y comunidades

- Buscar que los estudiantes sean partícipes del desarrollo y evolución de su criterio profesional desde la etapa inicial de la carrera

- Promover que los estudiantes alcancen mayores grados de madurez y coresponsabilidad en su propia formación

- Identificar como autoconstrucción la identidad profesional 
La percepción de los alumnos acerca de lo que será su inserción en el mercado de trabajo debe ser mucho más activa que en el pasado y no presuponer que en el mundo ocupacional de este siglo se encontrarán con problemas claramente definidos a los que se les solicitará, de modo explíccto, dar solución. Por el contrario, su formación debe presuponer, en este momento, una condición de permanente búsqueda y transformación así como una actitud propositiva.

Las empresas del pasado, como resultado de la Revolución Industrial, eran más fuertes mientras mayores recursos poseyeran: dinero, materiales y equipo, instalaciones, personal; puesto que éstos garantizaban su capacidad de producir y proporcionar bienes y servicios. En la actualidad, las empresas y las organizaciones en general tienen su mayor riqueza en las ideas. Así, un profesional del tercer milenio es más útil y produce más para sí mismo y para la sociedad en la medida en la que procese la información disponible en torno a una situación dada, y la transforme en ideas creativas que mejoren las condiciones existentes.

Las ventajas de estar viviendo en la sociedad del conocimiento merecen ser consideradas con cuidado. No es el acceso a la información per se la que producirá profesionales altamente creativos, socialmente responsables y realizados mediante su desempeño laboral. Se trata de que el sistema educativo en el nivel superior se dirija hacia apoyar la formación de cada uno de sus integrantes facilitando que se acceda a la información y se le analice desde diversas ópticas: mayor apertura hacia otras manifestaciones culturales y referentes sociales, comunicación inter e intradisciplinaria, destreza en el uso y aplicación de las nuevas herramientas tecnológicas, marco valoral y ético de las acciones, y, por supuesto el acento en la formación de actitudes de búsqueda, compromiso y tendencia al logro.

“... lo que más necesitan los jóvenes de sus escuelas y universidades no es una capacitación vocacional o una educación en disciplinas académicas tradicionales [únicamente] [...] necesitan [también] aprender lo necesario para hacer ofertas, para producir un valor especial para los [...] [beneficiarios de su idea] [...] empleadores potenciales, otros grupos dentro de la misma organización, el mercado o sus [propias] comunidades"7.

El maestro Carlos Llano, a este respecto, señala que a fin de lograr un modelo vanguardista de formación profesional se debería proporcionar, desde la escuela superior:

- Multivalencia idiomática

- Polivalencia cultural

- Aplicación continua de los avances cibernéticos

- Profundización de la dimensión ética de la enseñanza

- $\quad$ Interdisciplinariedad $^{8}$

Concretamente en este punto, Flores y Gray ${ }^{9}$ definen que la educación profesional en estos momentos requiere formar egresados que sean capaces de:

* Establecer identidades comprometedoras.

Sepan escuchar las preocupaciones de los otros y conectarse con ellos desde su historia particular, logrando así una verdadera integración para la satisfacción de necesidades comunitarias.

* Escuchar y atender conflictos, en apariencia irresolubles.

Identifiquen el concepto de la vida que motiva lo que los demás dicen y hacen. 
* Construir y mantener la confianza.

Administren compromisos y mantengan la confianza a fin de formar equipos sólidos. Capacitar a la gente para ver los beneficios de establecer acuerdos no trillados, imaginárselos y negociarlos unos con otros especialmente cuando comparten conflictos y se encuentran en lados opuestos.

* Ejercer el poder

Se encuentre entrenada para leer al mundo e identificar cómo y dónde reside el poder. Conciba el poder como el arte de construir relaciones novedosas hacia valores que le importan a la mayoría de la gente de una comunidad más que como creación de barreras. Gente que sea constructora activa de una identidad para sí misma.

\section{Formación del carácter}

Un lugar especial merece el punto relacionado con la formación del carácter. Es innegable que, como resultado de las grandes transformaciones económicas que han impactado el mercado laboral, han tenido lugar tanto recortes masivos de personal como dificultades para recontratarse, a causa de la elevación de los requisitos académicos y la demanda de personal profesional con un espectro de formación más amplio. Esto ha traído como consecuencia en las personas reacciones depresivas y de resentimiento dirigido hacia cualquier instancia que se considere responsable de tal estado de cosas: “...los ejecutivos de las compañías, los líderes políticos, los accionistas, los ricos, los pobres, los educadores, [...] [los] padres... ${ }^{10}$.

Recientemente, el responsable del sector salud de nuestro país, dio a conocer el alarmante dato de que en poco menos de dos décadas se prevé como uno de los más importantes problemas de salud pública, la depresión y sus efectos entre la población adulta y la perteneciente a la tercera edad. De ahí la importancia de enfatizar desde la educación el fortalecimiento del carácter como medida tanto preventiva frente a las potencialmente adversas condiciones de vida, como también, y sobre todo, con la mira de hacer frente al riesgo y transformarlo en oportunidad.

Como otro ejemplo de lo que se está llevando a cabo en la educación, ahora en el ámbito laboral, cito a continuación lo que se considera objetivo de capacitación y desarrollo para el personal de una gran empresa de influencia mundial:

$\begin{array}{cll}\text { S } & \text { (Skill) } & \begin{array}{l}\text { Habilidades } \\ \text { Tecnológicas }\end{array} \\ \text { M } & \text { (Management) } & \begin{array}{l}\text { Capacidad } \\ \text { Directiva }\end{array} \\ \text { I } & \text { (International) } & \begin{array}{l}\text { Visión } \\ \text { Planetaria }\end{array} \\ \text { L } & \text { (Language) } & \begin{array}{l}\text { Capacidad } \\ \text { de Comunicación }\end{array} \\ \text { E } & \text { (Effort) } & \begin{array}{l}\text { Capacidad de } \\ \text { Esfuerzo }\end{array}\end{array}$

Este objetivo de comunicación es el resultado del desarrollo de un anagrama que en el idioma inglés significa: SONRÍA ya que la primera cualidad que debe adquirirse frente al futuro pertenece no fundamentalmente a los conocimientos, o por lo menos no a los conocimientos por sí solos, sino al carácter. Hemos de afrontar con buena disposición, las dificultades que tenemos por delante.

El hecho de que sea posible, desde la educación, promover actitudes de fortaleza frente al riesgo y elevar la autoestima para 
asumir el reto, en lugar de aquellas que implican derrota anticipada, conformismo, cinismo o desilusión, constituye una ventaja. El empoderamiento de los individuos que se consideran a sí mismos capaces de encarar las dificultades les hace identificar nuevas oportunidades, mientras que la falta de confianza derivada del resentimiento, sólo les provoca inmovilidad y pérdida de confianza, de tiempo y de otros recursos ${ }^{12}$.

Del énfasis que se otorgue a la formación y al desarrollo integral de las personas depende el futuro. La educación formal y sistemática que ofrece la escuela no debe estar divorciada del principio de aprendizaje de búsqueda y mejoramiento de condiciones que implica al sujeto total, como característica intrínsecamente humana, el aprendizaje creativo, altamente satisfactorio y el cual, orientado de manera correcta, acompaña a las personas a lo largo de toda su vida. El concepto de ser humano educado debe estar más cerca de aquél que comprende mejor la realidad que le circunda y participa decidida y entusiastamente en la transformación de la misma a fin de hacerla más armónica para él y los otros, a partir del diálogo, el respeto, la convivencia pacífica y la defensa de la verdad.

\section{Conclusión}

La educación en todos los niveles, pero sobre todo la profesional, por las razones apuntadas antes, no puede seguirse aferrando a formas de organización que preparaban para actuar en condiciones que ya no existen ni existirán más, en el mundo del futuro. El resultado de no considerar lo que ha cambiado puede ser el de garantizar el fracaso.

La formación profesional, en tanto educación integral, debe incidir en el desarrollo de personalidades asertivas. Debe enfatizar el cultivo de disposiciones hacia el logro, la innovación, la búsqueda de alternativas y el valor para encarar los riesgos del cambio con iniciativa.

El aula universitaria debe tener cada vez más las características de un foro de análisis y reflexión colectiva, de aclaración de dudas y evaluación en conjunto, guía experta, comunicación e intercambio de los avances individuales, por equipos y por grupo. Considerarse un punto de encuentro y comunicación donde la formación profesional parta de un plan fundamental de desarrollo educativo concebido desde las instancias institucional y docente, en el cual el estudiante no sólo sea co-responsable sino motor, y por su parte el sistema educativo en su conjunto facilite, incentive y promueva el aprendizaje autónomo, reconozca, socialice y certifique sus logros.

Desde la escuela superior es menester enfatizar en el desenvolvimiento completo de la persona como autoconstrucción y crecimiento ininterrumpido a través del proceso de adquirir conocimientos, habilidades, actitudes y valores individual y socialmente útiles.

Frente a estas nuevas reglas del juego, el imperativo es la formación de profesionales que se perciban a sí mismos capaces de afrontar los retos del presente y no deprimidos por el resentimiento frente a la pérdida del pasado. La tarea educativa en este caso será intervenir en la formación de jóvenes con características tales, que su reacción frente al cambio en lugar de ser de desamparo y paralizante sea de iniciativa, estimulante, positiva y de compromiso.

Habrá que reflexionar en que el espíritu humano es en esencia el mismo que ha permitido durante todos estos siglos la transformación de la naturaleza para evolucionar como especie e inventar la cultura. 


\section{Notas}

1. Cfr. Flores, F. Creando organizaciones para el futuro. México, Dolmen. Y también Flores, F. Inventando la empresa del siglo XXI. Madrid, Hacheté.

2. Cfr. Flores, F. Creando organizaciones para el futuro. México, Dolmen. Y también Flores, F. Inventando la empresa del siglo XXI. Madrid, Hacheté.

3. Cfr. Barros, O. Reingeniería de procesos de negocios. Un planteamiento metodológico. México, Dolmen, 1999.

4. Gago Huguet, A. y otros. Tendencias actuales de la educación superior, p. 264.

5. $\quad$ Flores, F. y J. Gray. La vida wired. El trabajo en el ocaso de las carreras $3^{\underline{a}}$ parte, p. 88.

6. Durán Ramos, T. Hacia la caracterización del modelo educativo..., p. 83-84.

7. Flores, F. y J. Gray. Op. Cit, p. 88.

8. Llano Cifuentes, C. Empresa y universidad en el futuro, p. 21.

9. $\quad$ Flores, F. y J. Gray. Op. Cit., p. 90-93.

10. Ibídem, 94 .

11. Llano Cifuentes, C. Op. Cit., 14-15.

12. Porcentaje de jóvenes norteamericanos entre los 18 y los 29 años de edad que están de acuerdo en que Tal y como están las cosas a los de mi generación les resulta más difícil vivir desahogadamente que a los de las generaciones anteriores 65\% Revista TIME 16 de julio de 1990, citado por Coupland, D. C. Generación $X$, p. 255.

\section{Referencias bibliográficas}

Asociación Nacional de Universidades e Instituciones de Educación Superior. La educación superior en el siglo XXI. Líneas estratégicas de desarro- llo. Una propuesta de la ANUIES. México, ANUIES, 2000. 260 p.

Barros, O. Reingeniería de procesos de negocios. Un planteamiento metodológico. México, Dolmen, 1999.

Coupland, D. C. Generación X. Tr. Mariano Antolín Rato. Barcelona, Ediciones B, 2000. 256 p. (Colección Tiempos Modernos).

Delors, J. La educación encierra un tesoro. Informe a la UNESCO de la Comisión Internacional sobre la Educación para el siglo XXI. México, Correo de la UNESCO, 1997. 302 p.

Durán Ramos, T. "Hacia la caracterización del modelo educativo con el que hoy se forma a los estudiantes de Pedagogía de la Facultad de Filosofía y Letras”. En: Memoria del Coloquio: La pedagogía hoy. México, UNAM, Facultad de Filosofía y Letras, 1994. pp. 79-84.

Flores, F. Creando organizaciones para el futuro. México, Dolmen, 1998.

Inventando la empresa del siglo XXI. Madrid, Hacheté, 1999.

. J. Gray. "La vida wired. El trabajo en el ocaso de las carreras". Tr. Araceli Fernández. En: NEXOS núms. 270, 271 y 272 ; jun.-jul. y ago. del 2000.

Gago Huguet, A. y otros. Tendencias actuales de la educación superior en el mundo. México, UNAM, 1986. 401 p.

Gardner, H. La educación de la mente y el conocimiento de las disciplinas. Buenos Aires, Piados. 2000. 
Llano Cifuentes, C. Empresa y universidad en el futuro. México, Universidad Panamericana, Facultad de Filosofía, Departamento de Filosofía Práctica. 34 p. (Humanismo y empresa, 15).
UNESCO, "Declaración Mundial sobre la Educación Superior en el Siglo XXI: Visión y Acción” 1998. http://www.unesco.org/education/educprog/wche/declaration_spa.htm 